\title{
Characterization of the Physiological Turnover of Native and Inactivated Cytochromes P450 3A in Cultured Rat Hepatocytes: A Role for the Cytosolic AAA ATPase p97? ${ }^{\dagger}$
}

\author{
Saadia Faouzi ${ }^{\infty}$, Katalin F. Medzihradszky $₫$, Colleen Hefner§, Jacquelyn J. Maher§, and Maria \\ Almira Correia $£^{\prime *}$ \\ $\infty$ Department of Cellular \& Molecular Pharmacology and The Liver Center, University of California, San \\ Francisco, CA 94158
}

IDepartment of Pharmaceutical Chemistry and The Liver Center, University of California, San Francisco, CA 94158

§Department of Medicine and The Liver Center, University of California, San Francisco, CA 94158

£Departments of Cellular \& Molecular Pharmacology, Pharmaceutical Chemistry, Biopharmaceutical Sciences, and Medicine and The Liver Center, University of California, San Francisco, CA 94158

\begin{abstract}
Mammalian hepatic cytochromes P450 (P450s) are endoplasmic reticulum (ER)-anchored hemoproteins engaged in the metabolism of numerous xeno- and endobiotics. P450s exhibit widely ranging half-lives, utilizing both autophagic-lysosomal (ALD) and ubiquitin-dependent $26 \mathrm{~S}$ proteasomal (UPD) degradation pathways. Although suicidally inactivated hepatic CYPs 3A and "native" CYP3A4 in S. cerevisiae are degraded via UPD, the turnover of native hepatic CYPs 3A in their physiological milieu has not been elucidated. Herein, we characterize the degradation of native, dexamethasone-inducible CYPs 3A in cultured primary rat hepatocytes, using proteasomal (MG-132 and MG-262) and ALD [ $\left[\mathrm{NH}_{4} \mathrm{Cl}\right.$ and 3-methyladenine (3-MA)] inhibitors to examine their specific degradation route. Pulse-chase cum immunoprecipitation analyses revealed a basal 52\% ${ }^{35} \mathrm{~S}-\mathrm{CYP} 3 \mathrm{~A}$ loss over $6 \mathrm{~h}$, which was stabilized by both proteasomal inhibitors. By contrast, no corresponding CYP3A stabilization was detected with either ALD inhibitor $\mathrm{NH}_{4} \mathrm{Cl}$ or 3-MA. Furthermore, MG-262-induced CYP3A stabilization was associated with its polyubiquitylation, thereby verifying that native CYPs $3 \mathrm{~A}$ were also degraded via UPD. To identify the specific participants in this process, cellular proteins were crosslinked in situ with paraformaldehyde (PFA) in cultured hepatocytes. Immunoblotting analyses of CYP3A immunoprecipitates after PFA-crosslinking revealed the presence of p97, a cytosolic AAA ATPase instrumental in the extraction and delivery of ubiquitylated ER proteins for proteasomal degradation. Such native CYP3A-p97 interactions were greatly magnified after CYP3A suicidal inactivation (which accelerates UPD), and/or proteasomal inhibition, and were confirmed by proteomic and confocal immunofluorescence microscopic
\end{abstract}

\footnotetext{
${ }^{\dagger}$ Supported by NIH grants GM44037 (MAC) and DK26506 (MAC), RR012961 (KFM) and NIH grant P30DK26743 (Liver Core Center Cell and Tissue Biology Core).

*Corresponding Author: M. A. Correia Dept. of Cellular and Molecular Pharmacology, Mission Bay Campus, Genentech Hall 600 16th Street, N572F/Box 2280 University of California San Francisco, CA 94158-2280 415-476-3992 (TEL) 415-476-5292 (FAX) e-mail: almira.correia@ucsf.edu.

Supporting Information: MS/MS data obtained from the proteomic analyses of CYP3A-protein crosslinked complexes after subjection to SDS-PAGE, sequential gel-slicing of each relevant lane, and in situ tryptic digestion followed by LC-MS/MS analyses are provided. The proteins were identified from more than 1 peptide, but only 1 representative spectrum has been provided for each in the supplementary material. This material is available free of charge via the Internet at http://pubs.acs.org
} 
analyses. These findings clearly reveal that native CYPs 3A undergo UPD and implicate a role for p97 in this process.

The hepatic hemoproteins cytochromes P450 (P450s) ${ }^{1}$ are key enzymes in the oxidative metabolism of various endobiotics and xenobiotics. Each P450 is anchored to the endoplasmic reticulum (ER) membrane via its hydrophobic $\leq 27$ residue long N-terminus, with the bulk of its catalytic domain exposed to the cytosol, and thus is an excellent prototype of an integral monotopic ER-protein. All ER-anchored P450s, albeit differing in their primary sequence, active site structure and substrate selectivity exhibit this ER-topology and tertiary structural fold. Thus it is surprising that $\mathrm{P} 450$ s exhibit highly variable protein half-lives ranging from a $\mathrm{t}_{1 / 2}$ of 7 and $14 \mathrm{~h}$ for substrate-free CYP2E1 and CYP3A23, respectively, to half-lives $>20 \mathrm{~h}$ for CYPs 2B1, 2C6 and substrate-liganded CYP2E1 (Reviewed in ${ }^{1}$ ). Two major cellular pathways for protein degradation exist in eukaryotes from yeast to mammals: The relatively slow autophagiclysosomal degradation (ALD) $(2,3)$, and the considerably faster ERassociated degradation (ERAD), a process that involves ER-protein extraction and its subsequent ubiquitin (Ub)-dependent 26S proteasomal degradation (UPD) $\left(4^{-6}\right)$. Studies of mammalian hepatic P450 degradation in intact animals, cultured hepatocytes, cell lines and yeast, have indicated that consistent with their relatively longer half-lives, native P450s such as CYPs 2B1, CYP2C11 and substrate-bound CYP2E1 are largely turned over by the slower ALD process $\left(7^{-12}\right)$. By contrast, after chemical-induced structural and functional inactivation, most P450s including CYPs 3A ${ }^{2}$, CYP2B1, CYP2C11 and CYP2E1 in common with most structurally abnormal and/or misfolded ER proteins, are rapidly degraded via ERAD $\left(13^{-26}\right)$. These findings attest to a mechanistically highly versatile disposal process, possibly entailing structural determinants or degradation signals "degrons", posttranslational modifications, and/ or chaperones for sorting P450s into either ALD or ERAD/UPD.

Both ALD and ERAD/UPD pathways of protein degradation are evolutionarily highly conserved in eukaryotes from yeast to mammals $\left(4^{-7}, 27,28\right)$. Indeed, our studies of P450 degradation in the yeast Saccharomyces cerevisiae indicate that consistent with the rat liver findings, heterologously expressed "native" CYPs 2B1 and 2C11 were degraded via ALD

\footnotetext{
${ }^{1}$ Abbreviations used:

adenylate kinase (AK)

autophagic lysosomal degradation (ALD)

cytochromes P450 (P450s)

3,5-Dicarbethoxy-2,6-dimethyl-4-ethyl-1,4-dihydropyridine (DDEP)

endoplasmic reticulum (ER)

ER-associated degradation (ERAD)

high molecular mass (HMM)

immunoblotting (IB)

LC/MS/MS

3-methyladenine (3-MA)

paraformaldehyde (PFA)

transitional endoplasmic reticulum (TER) ATPase

troleandomycin (TAO),

ubiquitin (Ub)

Ub-conjugating enzyme (E2)

ubiquitin-dependent $26 \mathrm{~S}$ proteasomal (UPD) degradation

Ub-ligase (E3)

VCP (valosin-containing protein),

William's E (WE)

2 Although the antibody was generated against purified rat liver CYP3A23, the major hepatic dexamethasone (DEX)-inducible CYP3A isoform, it recognizes all CYP3A isoforms. Since the hepatocytes were derived from DEX-treated male rat liver, we could not distinguish between individual CYP 3A2, 3A9, 3A23 and 3A18 isoforms, and thus have collectively termed them CYPs 3A. Apparently, in DEXtreated male rat liver, the allelic variant CYP3A1 is either absent or a minor component, while CYP3A23, 3A18, 3A2 and 3A9 mRNA expression respectively account for $73,17,6$ and $1 \%$ of the total CYP3A transcripts $(56,57$ ). In untreated male rat liver, CYP3A23 is similarly the predominantly expressed CYP3A transcript (67\%), followed by CYP3A2 (30\%), and the remainder consists of very minor albeit equivalent levels of CYPs 3A18 and 3A9.
} 
$(11,12)$. In contrast, similarly expressed "native" CYP3A4 was degraded in a classical ERAD/ UPD pathway, as confirmed by the involvement of the following bona fide ERAD components: Cytosolic Ub-conjugating enzyme Ubc7p and its ER-membrane anchor Cue1p, the essential proteasomal 19S cap subunit Hrd2p, as well as the AAA ATPase chaperone complex Cdc48pUfd1p-Hrd4p $(25,26)$. Yeast Cdc48p is homologous to the mammalian p97 or VCP (valosincontaining protein), whose chaperone function also requires complexation with Ufd1p and Npl4p, the Hrd4p homolog $\left(28^{-35}\right)$. The Cdc48p chaperone machinery is required for the retrotranslocation/extraction of lumenal and integral proteins from the ER before their delivery to the cytosolic $26 \mathrm{~S}$ proteasome for degradation $\left(28^{-35}\right)$. To exclude any concerns that such CYP3A4 ERAD-targeting was due to the structural misfolding of this human protein in yeast, we sought to examine the degradation of CYPs $3 \mathrm{~A}$ in a more natural physiological milieu: Rat hepatocytes cultured in a collagen Type I-Matrigel sandwich, that maintains even difficult to preserve hepatic $\mathrm{P} 450$ processes (i.e. CYP2B1 induction and function) near normal $\left(36^{-38}\right)$.

Our findings described herein indicate that in cultured rat hepatocytes, "native" CYPs $3 \mathrm{~A}$ in common with the suicidally inactivated CYP3A species are clearly degraded via ERAD/UPD just as CYP3A4 is in S. cerevisiae $(25,26)$. Furthermore, in situ chemical crosslinking in cultured hepatocytes with paraformaldehyde (PFA), followed by proteomic analyses of the immunoprecipitated CYP3A-crosslinked protein complexes coupled with confocal immunofluorescence microscopic (CIFM) analyses revealed that this CYP3A ERAD apparently also involves the AAA ATPase p97, the mammalian Cdc48p homolog.

\section{Materials and Methods}

\section{Materials}

Bovine serum albumin (BSA), insulin-transferrin-selenium (ITS), L-glutamine, penicillinstreptomycin, phenylmethylsulfonyl fluoride (PMSF) and William's E (WE) medium were obtained from Invitrogen (Carlsbad, CA). Methionine- and cysteine-free WE medium was prepared by the UCSF Cell Culture Facility. Ammonium chloride $\left(\mathrm{NH}_{4} \mathrm{Cl}\right)$, dexamethasone (Dex), paraformaldehyde (PFA), troleandomycin (TAO), and 3-methyladenine (3-MA) were purchased from Sigma-Aldrich (St. Louis, MO). Aprotinin, E-64 and leupeptin were from Roche Diagnostics (Indianapolis, IN). 3,5-Dicarbethoxy-2,6-dimethyl-4-ethyl-1,4dihydropyridine (DDEP) was synthesized as previously described (39). Matrigel was purchased from BD Biosciences (Bedford, MA). MG-132 and MG-262 were obtained from Boston Biochem. EasyTag Express ${ }^{35}$ S-Protein Labeling Mix was obtained from Perkin Elmer (Boston, MA). Mouse anti-p97 IgGs used for Western immunoblotting (IB) was from RDI/ Fitzgerald Industries International, Inc (Concord MA), Alexa Fluor 488 anti-rabbit IgGs, Alexa Fluor 568 anti-mouse IgGs and mouse anti-p97 antibodies used for immunostaining were purchased from Molecular Probes (Eugene, OR) and BD Transduction Laboratories (San Jose, CA). ToxiLight cytotoxicity assay kit was purchased from Cambrex Bio Science (Rockland, ME)

\section{Animals}

Male Sprague-Dawley rats (225-250g) were purchased from Charles River Laboratories (Wilmington, MA). Rats were housed at the UCSF Animal Care Facility, fed and given water ad libitum and handled according to IACUC guidelines, until killing.

\section{Hepatocyte Isolation and Culture}

Hepatocytes were isolated from male Sprague-Dawley rats by in situ perfusion of the liver with collagenase (liver digest medium) and purified by centrifugal elutriation as previously described $(38,40)$. Hepatocytes $\left(3 \times 10^{6}\right)$ were seeded onto $35 \mathrm{~mm}$ Permanox culture dishes pre-coated with type I Collagen. Cells were cultured in WE medium containing ITS, $0.1 \mu \mathrm{M}$ 
Dex, $50 \mathrm{U} / \mathrm{ml}$ penicillin/streptomycin, $2 \mathrm{mM} \mathrm{L}$-glutamine, and $0.1 \%$ BSA. Cells were overlaid with $0.25 \mathrm{mg} / \mathrm{ml}$ Matrigel $2 \mathrm{~h}$ after plating as previously described (36). Cells were maintained for 2 days with a daily change of medium then induced with $5 \mu \mathrm{M}$ Dex for 3 days. Treatments were started on the fifth day of culture. Cells were treated with vehicle (ethanol or DMSO), Dex $(5 \mu \mathrm{M})$, autophagic-lysosomal inhibitors $\mathrm{NH}_{4} \mathrm{Cl}(20 \mathrm{mM})$ and/or 3-MA $(5 \mathrm{mM})$ or proteasomal inhibitors MG-132 $(20 \mu \mathrm{M})$ or MG-262 $(10 \mu \mathrm{M})$, with or without $100 \mu \mathrm{M}$ DDEP. Cells were harvested for the preparation of cell lysates. For the TAO experiments, on the $5^{\text {th }}$ day of culture in a WE medium containing Dex $(5 \mu \mathrm{M})$, hepatocytes were ${ }^{35}$ S-pulse-labeled at $0 \mathrm{~h}$, chased with an excess amount of unlabeled methionine $(10 \mathrm{mM})$ for $1 \mathrm{~h}$, after which fresh medium containing TAO was added and cells harvested after 1, 24, or $30 \mathrm{~h}$ of labeling. When the effect of proteasomal or ALD inhibitor on TAO-complexed CYP3A was examined, the agent was added at $24 \mathrm{~h}$ to TAO-treated hepatocytes, and the cells harvested $6 \mathrm{~h}$ later (at $30 \mathrm{~h})$.

\section{Cytotoxicity Assay}

Cytotoxicity was examined using ToxiLight cytotoxicity assay as per the manufacturer's instructions exactly as described (38). The assay consists of chemiluminescent detection of adenylate kinase (AK) that is released into the medium by damaged hepatocytes using the reconstituted $\mathrm{AK}$ detection reagent. A positive control corresponding to $100 \%$ cytotoxicity was obtained by monitoring the AK released from freeze-thawed cells. $\mathrm{H}_{2} \mathrm{O}_{2}$-treatment $(10 \mathrm{mM})$ of cell cultures was also included as a positive control, but found to be inefficient in releasing cellular AK content compared with repeated freeze-thaw cycles or cell lysis by sonication.

\section{${ }^{35}$ S-Pulse-Labeling of Newly Synthesized P450 Proteins}

Hepatocytes were preincubated in a methionine- and cysteine-free WE medium at $37^{\circ} \mathrm{C}$ for $1 \mathrm{~h}$. The cells were then pulse-labeled with L- $\left[{ }^{35} \mathrm{~S}\right]$ methionine/cysteine $(60 \mu \mathrm{Ci})$ for $1 \mathrm{~h}$ and then chased with an excess amount of unlabeled methionine $(10 \mathrm{mM})$. Labeled cells were harvested at $0 \mathrm{~h}, 1 \mathrm{~h}$ or $6 \mathrm{~h}$ in lysis buffer $(50 \mathrm{mM}$ Tris $\mathrm{HCl} \mathrm{pH} 8,0.5 \mathrm{mM}$ EDTA, $5 \mathrm{mM}$ EGTA, $1 \% \mathrm{SDS}, 0.2 \mu \mathrm{M}$ aprotinin, $15 \mu \mathrm{M}$ leupeptin, and $10 \mu \mathrm{M} \mathrm{E}-64)$.

\section{Paraformaldehyde (PFA) Crosslinking}

Cells were washed with PBS and incubated with $2.5 \%$ PFA for $15 \mathrm{~min}$ at $37^{\circ} \mathrm{C}$. Crosslinking was stopped with $0.1 \mathrm{M}$ glycine (41). Cells were harvested in lysis buffer described above. Crosslinking was reversed by boiling the samples in 5\% SDS-PAGE buffer containing 10\% glycerol, $40 \mathrm{mM}$ Tris, pH 6.8, containing DTT $(50 \mathrm{mM})$ and 2.5\% $\beta$-mercaptoethanol (41).

\section{${ }^{35}$ S]CYP3A Immunoprecipitation}

Total protein $(300 \mu \mathrm{g})$ was immunoprecipitated with goat anti-CYP3A IgGs $(250 \mu \mathrm{g})$. Immunoprecipitation was performed as previously described (23). Immunoprecipitates were subjected to SDS-PAGE on a precast $4-20 \%$ gradient gel. Gels were fixed and then dried on filter paper before PhosphorImager analyses.

\section{IB Analysis of Ubiquitylated CYPs 3A}

CYP3A ubiquitylation was assessed by Western IB analyses of CYP3A immunoprecipitates with rabbit anti-Ub IgGs as previously described (23).

\section{Immunofluorescence Staining}

After PFA-crosslinking, cells were fixed in methanol for $15 \mathrm{~min}$ at $-20^{\circ} \mathrm{C}$ and incubated for 1 $\mathrm{h}$ with $1 \%$ normal goat serum. Cells were then incubated with rabbit anti-CYP3A and mouse anti-p97 antibodies for $1 \mathrm{~h}$ at room temperature. After washing with PBS, cells were incubated 
with Alexa Fluor 488 goat anti-rabbit and Alexa Fluor ${ }^{\circledR} 568$ goat anti-mouse antibodies. Cells were observed with a Leica TCS NT Laser Scanning Confocal Microscope.

\section{LC-MS/MS Analysis}

Proteins coimmunoprecipitated with CYP3A were identified using mass spectrometric sequencing analysis. To exclude non-specific interactions and/or spurious contaminants, mock immunoprecipitations were conducted in parallel that replaced the Melon ${ }^{\mathrm{TM}}$ gel purified goat anti-CYP3A IgG either with preimmune goat IgG or non-purified goat anti-CYP3A IgG. Immunoprecipitations devoid of hepatocyte lysates were also included in parallel as controls. Crosslink-reversed immunoprecipitates were fractionated by SDS-PAGE and stained with Coomassie Blue. Nine bands from each lane were sequentially excised, reduced, alkylated with iodoacetamide, and in-gel digested with side-chain protected porcine trypsin (http://ms-facility.ucsf.edu/ingel.html). An aliquot from each digest was fractionated by reversed-phase HPLC using an Ultimate Pump/Famos autosampler system (Dionex/LC Packings), a homemade nanocolumn $(\mathrm{C} 12,75 \mu \mathrm{m} \times 150 \mathrm{~mm})$ and a LTQ ion trap (Thermo Finnigan) used as a detector/analyzer in a data-dependent fashion: Survey mass acquisitions were followed by a $10 \mathrm{Da}$ wide "zoom-in" scan that allowed higher resolution mass and charge determination of the most abundant ion, and if it proved multiply charged a CID acquisition followed. Data files were processed with Mascot distiller, and database searches were performed using an in-house Mascot server. The entire SwissProt database was searched. Search parameters: Only tryptic peptides were considered, and one missed cleavage was permitted; mass accuracy was $2 \mathrm{Da}$ for the precursor ions and $0.8 \mathrm{Da}$ for the peptide fragments; carbamidomethylation of Cys residues was considered as a fixed modification; Met oxidation, protein $\mathrm{N}$-acetylation and cyclization of $\mathrm{N}$-terminal Gln residues were permitted as variable modifications. Peptides with probability scores $\mathrm{p}<0.05$ were accepted.

\section{Results}

\section{Characterization of the physiologic turnover of native CYPs $3 \mathrm{~A}$ in cultured rat hepatocytes}

A primary rat hepatocyte culture model was optimized for studies of hepatic P450 degradation. This system is based on pulse-chase analyses in rat hepatocytes cultured on a collagen type I substratum with a Matrigel overlay. We have used this system to characterize the physiological degradation of "native" Dex-inducible CYPs 3A (Fig. 1A-C). Immunoprecipitation analyses with specific goat anti-rat liver CYP3A23 antibodies revealed a time-dependent loss of newly synthesized ${ }^{35} \mathrm{~S}$-labeled CYP3A over $6 \mathrm{~h}$ which was completely or partially blocked by inclusion of the proteasomal inhibitor MG-262 or MG-132 3 , respectively, but not of the lysosomal inhibitor $\mathrm{NH}_{4} \mathrm{Cl}$ or the autophagosomal inhibitor 3-MA (Fig. 1A).

Accordingly, ${ }^{35} \mathrm{~S}$-radiometric quantitation of these CYP3A immunoprecipitates revealed that of the initial $(0 \mathrm{~h}){ }^{35} \mathrm{~S}-\mathrm{CYP} 3 \mathrm{~A}$ content, $73.3 \pm 39.2 \%$ remained at $1 \mathrm{~h}$, falling to $38.3 \pm 16.1 \%$ at $6 \mathrm{~h}$ (Fig. 1A). This 50-60\% loss of the initial ${ }^{35} \mathrm{~S}-\mathrm{CYP} 3 \mathrm{~A}$ content at $6 \mathrm{~h}$ was completely blocked by MG-262 and partially by MG-132, but not by either $\mathrm{NH}_{4} \mathrm{Cl}$ or 3-MA, thereby indicating that it was predominantly due to proteasomal degradation (Fig. 1B). When these ${ }^{35}$ S-CYP3A immunoprecipitates were subjected to SDS-PAGE/PhosphorImager analyses, a relative decrease of the $55 \mathrm{kDa}^{35} \mathrm{~S}$-CYP3A species was clearly detected at $\approx 1 \mathrm{~h}$,

\footnotetext{
${ }^{3}$ Both MG-132 and MG-262 target one of the catalytic 20 S proteasomal Thr residues. Although the MG-132 aldehyde moiety forms a covalent hemiacetal linkage with this Thr residue, this linkage can be hydrolyzed under physiological conditions, making proteasomal peptide aldehyde inhibitors reversible, competitive inhibitors. In addition, their propensity to oxidize to acids under physiological conditions also contributes to their lower metabolic stability. In contrast, MG-262 is a peptide boronate. Boronic acids have a high affinity for the Thr hydroxyl group and the relative strength of the boron-oxygen bond, coupled with much slower off-rates than those of peptide aldehydes, endow them with greater metabolic stability (58). Thus although classified as covalent, competitive and reversible inhibitors, this property in addition to their potency and selectivity, not only makes them superior to peptide aldehyde inhibitors, but also suitable for clinical use as one prototype (Velcade) has proved. This difference in stability, we believe accounts for the shorter duration of MG-132mediated inhibition of CYP3A degradation.
} 
and this loss was further enhanced at $6 \mathrm{~h}$. In parallel, an increase in high molecular mass (HMM ${ }^{35}$ S-CYP3A species was observed at $1 \mathrm{~h}$, subsiding thereafter at $6 \mathrm{~h}$. These findings are consistent with CYP3A protein ubiquitylation before its proteolytic degradation (Fig. 1C). Proteasomal inhibition by treatment with MG-262 or MG-132 greatly enhanced the detection of these HMM ${ }^{35} \mathrm{~S}-\mathrm{CYP} 3 \mathrm{~A}$ species particularly at $6 \mathrm{~h}$, while reducing the loss of the 55 $\mathrm{kDa}^{35} \mathrm{~S}-\mathrm{CYP} 3 \mathrm{~A}$ species at both time points ${ }^{4}$. In contrast, the autophagic/lysosomal inhibitors had minimal effect on either parameter. Indeed, parallel IB analyses of these ${ }^{35}$ S-CYP3A immunoprecipitates with rabbit anti-Ub IgGs yielded the characteristic HMM ladders of polyubiquitylated CYP3A, which were dramatically enhanced in the presence of proteasomal, but not autophagic/lysosomal inhibitors, with marked accumulation observed at $6 \mathrm{~h}$ relative to the vehicle-treated cultures (Fig. 1D). These findings thus indicated that the physiological turnover of native CYP3A in cultured rat hepatocytes occurs via UPD. The absence of any oxidative stress-induced cytotoxicity in this culture system that might have led to CYP3A inactivation and/or oxidative damage and consequent UPD, was excluded by the detection of minimal $(<10 \%)$ spillage of the cytosolic enzyme AK into the medium under the various treatment conditions (Fig. 2).

\section{TAO-mediated stabilization of native CYP3A degradation}

The effect of TAO, the specific CYP3A inactivator that quasi-irreversibly complexes its heme (via metabolic-intermediate "MI" complexes) was determined as follows: One h after ${ }^{35} \mathrm{~S}$ pulse-labeling, TAO $(10 \mu \mathrm{M})$ or DMSO (vehicle) was added to the medium and the cultures incubated for another $24 \mathrm{~h}$. Some of these $24 \mathrm{~h}$-labeled cultures were also further treated for a $6 \mathrm{~h}$ period with or without DMSO, MG-262 $(10 \mu \mathrm{M})$, and/or 3-MA $(5 \mathrm{mM}) / \mathrm{NH}_{4} \mathrm{Cl}(20 \mathrm{mM})$. Inclusion of TAO in the culture medium for 24 or $30 \mathrm{~h}$, extensively blocked CYP3A degradative loss, consistent with its reported ability to "induce" CYP3A via protein stabilization (Fig. 3A-B; ${ }^{42}$ ). Thus, it appears from the slight but appreciably increased CYP3A levels observed at $6 \mathrm{~h}$ of either MG-262 or 3-MA/ $\mathrm{NH}_{4} \mathrm{Cl}$ treatment, that in the presence of TAO, CYP3A was now targeted partly into UPD but also partly into ALD (Fig. 3B). This is in contrast to the non-TAO complexed CYP3A that is stabilized only by the proteasomal (UPD) inhibitor MG-262, but not by the ALD inhibitors 3-MA/ $\mathrm{NH}_{4} \mathrm{Cl}$. Interestingly, TAOcomplexation did not affect the polyubiquitylated CYP3A fraction detectable at any given time, as reflected by the nearly equivalent CYP3A ubiquitylation profiles in TAO-treated and untreated hepatocytes (Fig. 3C). However, although marked accumulation of polyubiquitylated CYPs 3A is observed after MG-262-mediated proteasomal inhibition (Fig. 3C), this was greatly reduced after TAO complexation (Fig. 3C). Thus, it appears that TAO-complexation reduced CYP3A ubiquitylation and consequently its flux through the UPD pathway, consistent with the concomitantly reduced CYP3A susceptibility to proteasomal degradation. On the other hand, as expected, little accumulation of polyubiquitylated CYP3A was detected when the ALD pathway was blocked by 3-MA/ $\mathrm{NH}_{4} \mathrm{Cl}$.

\section{Enhanced CYP3A ubiquitylation and degradation after DDEP-mediated suicide inactivation in cultured rat hepatocytes}

We have previously shown that both in intact rats and freshly isolated rat hepatocytes in suspension, DDEP rapidly inactivates CYPs 3A via heme-modification of the protein, which marks the DDEP-inactivated CYPs $3 \mathrm{~A}$ for UPD $(13,14,17,18,23)$. This is also true of cultured rat hepatocytes (Fig. 4A \& B). Immunoprecipitation coupled with SDS-PAGE/

PhosphorImager analyses of ${ }^{35}$ S-labeled CYPs 3A $1 \mathrm{~h}$ after DDEP treatment, reveals a marked

\footnotetext{
${ }^{4}$ At these concentrations, while clearly protecting CYP3A from proteasomal degradation as expected, neither MG-262 nor MG-132 showed any CYP3A mRNA suppressive effects and/or inhibition of CYP3A protein synthesis as reported by others $(59,60$ ). It is unclear whether this is due to the much lower inhibitor concentrations than previously used (59), or due to our hepatocyte culture in a Matrigelcollagen Type I sandwich rather than just a Matrigel substratum (60).
} 
loss of the $55 \mathrm{kDa}$ CYP3A species relative to that in the untreated control (Fig. 4A). Pretreatment with the proteasomal (MG-262, MG-132) but not lysosomal inhibitors (3-MA, $\mathrm{NH}_{4} \mathrm{Cl}$ ) led to an accumulation of $\mathrm{HMM}{ }^{35}$ S-CYP3A species (Fig. 4A). Parallel IB analyses of these CYP3A immunoprecipitates with rabbit anti-Ub IgGs revealed that these HMM CYP3A species were indeed polyubiquitylated, and this profile was greatly enhanced by proteasomal inhibition by either MG-262 or MG-132 (Fig. 4A). This model thus seemed quite suitable for the molecular dissection of the cellular proteins involved in this UPD process.

\section{PFA-crosslinking analyses for detection of cellular proteins involved in CYP3A UPD}

As an initial approach towards identification of the cellular participants in CYP3A ERAD, we employed in situ PFA crosslinking to trap the proteins interacting with CYPs 3A. This was carried out in the presence or absence of the proteasomal inhibitor MG-262, both with untreated cells (native CYP3A) and $1 \mathrm{~h}$ after DDEP-treatment when CYP3A inactivation is at its peak and ubiquitylation is expected to be greatly stimulated. PFA crosslinking has many advantages: (i) It stabilizes protein interactions through covalent bond formation, thereby enabling the detection of weak and/or transient protein-protein interactions in native cells and tissues; and (ii) PFA is water soluble, cell-membrane permeable and fast reacting, and the crosslinking reversible by boiling in SDS-PAGE loading buffer. PFA-crosslinking was followed by CYP3A immunoprecipitation, SDS-PAGE of the immunoprecipitated complexes coupled with tryptic digestion and proteomic analyses or IB analyses, before and after reversal of the PFA-induced protein crosslinks. Each electrophoresed gel lane was sequentially sliced, and the protein bands reduced, carbamidomethylated and subjected to in-gel tryptic digestion, followed by datadependent liquid chromatography tandem mass spectrometry (LC-MS/MS) analyses of the tryptic peptides and automated database mining for protein identification, using Mascot (Matrix Science) search against the entire Swiss Protein Database.

The PFA-crosslinked CYP3A immunoprecipitates from untreated or DDEP- and/or MG-262treated hepatocytes, migrated to a region > $204 \mathrm{kDa}$, just below the top of the gel. The LC/MS/ MS profiles from all these culture groups, irrespective of treatment revealed the presence of CYP3A, ubiquitin and transitional endoplasmic reticulum (TER) ATPase also known as p97 or VCP (valosin-containing protein), the mammalian homolog of yeast Cdc48p. These proteins were absent in corresponding tryptic digests from parallel mock immunoprecipitation controls with pre-immune IgGs or controls devoid of lysates analyzed in parallel, thereby confirming the specificity of the immune interactions. However, no other plausible UPD-participant such as an Ub-conjugating enzyme (E2) or an Ub-ligase (E3) could be detected in these immunoprecipitated CYP3A complexes.

IB analyses of these CYP3A immunoprecipitate aliquots with mouse anti-rat p97 IgGs confirmed the presence of p97 crosslinked with CYP3A in untreated hepatocyte cultures, which were greatly magnified after DDEP- and/or MG-262-treatment, consistent with enhanced CYP3A ubiquitylation and accumulation of this ubiquitylated species on proteasomal inhibition (Fig. 5A). SDS-PAGE/proteomic analyses of reversed PFA-crosslinked complexes revealed the same partners that now migrated according to their relative electrophoretic masses. IB analyses of these CYP3A complexes with anti-p97 IgGs confirmed the presence of CYP3Acoimmunoprecipitated p97 in untreated hepatocytes, which was markedly increased by DDEPtreatment. This increase was further augmented by combined DDEP/MG-262-treatment. Parallel confocal immunofluorescence microscopic (CIFM) analyses of in situ PFA-X-linked DDEP- or DDEP/MG-262-treated hepatocytes using anti-CYP3A and anti-p97 IgGs, verified their colocalization and confirmed their in vivo association (Fig. 5B). This colocalization was greatly increased by combined DDEP/MG-262 treatment, consistent with the findings of the proteomic analyses. These findings thus indicated that in rat hepatocytes, CYP3A degradation is also associated with p97, a homolog of Cdc48p required for CYP3A4 ERAD in yeast (26). 


\section{Discussion}

Together our findings document that native CYPs $3 \mathrm{~A}$ are also physiologically targeted to UPD rather than ALD in cultured primary rat hepatocytes. We also document that this propensity for CYP3A UPD was considerably reduced by TAO, the relatively specific CYP3A quasiirreversible mechanism-based substrate inactivator, which stabilized the enzyme. Apparently, TAO-complexation conformationally stabilizes the CYP3A protein and renders it less susceptible to ubiquitination, possibly by concealing critical target Lys-residues and/or structural degrons required for its recognition by a specific Ub-ligase. Indeed, after TAOtreatment, a fraction of CYP3A was diverted into ALD as reflected by its newly acquired partial susceptibility to ALD inhibitors. This is consistent with the protein stabilization and dramatic half-life prolongation of the TAO-complexed CYP3A previously noted in intact rats and rat hepatocytes (42). Furthermore, just as in intact rat liver and freshly isolated rat hepatocytes $(13,14,17,18,23)$, this CYP3A UPD in rat hepatocytes is also greatly accelerated by DDEPinactivation which structurally damages the CYP3A protein by irreversible heme-modification and through subsequent ubiquitylation marks it for rapid cellular disposal by the $26 \mathrm{~S}$ proteasome. Our finding that physiologically, the native CYP3A protein is also similarly targeted to the UPD pathway raises the issue of whether it too, in the course of its normal oxidative/redox function, incurs structural damage similar to that of the DDEP-inactivated CYP3A protein. Indeed, several independent albeit complementary observations reinforce the notion that irreversible heme-modification of the protein also targets the native CYPs $3 \mathrm{~A}$ to UPD: (i) Irreversible heme-modification of the CYP3A protein occurs physiologically (13, 14). (ii) Such modification of the CYP3A protein is also observed after its $\mathrm{H}_{2} \mathrm{O}_{2}$-mediated inactivation, and $\mathrm{H}_{2} \mathrm{O}_{2}$ is a well-recognized byproduct of futile oxidative cycling of $\mathrm{P} 450 \mathrm{~s}$ in the absence of a substrate ligand. (iii) The short-lived CYPs $3 \mathrm{~A}$ are notoriously prone to high oxidative cycling in the absence of substrates, and substrate ligation (i.e. TAO-complexation) prolongs their half-life $(11,42-46$ ). (iv) A marked increase in CYP3A10/11 content is observed after conditional deletion of hepatic $\mathrm{P} 450$ reductase in mice (47). This, together with the observation that irreversible inactivation of $\mathrm{P} 450$ reductase by diphenylene iodonium also markedly (> 6-fold) extends the half-life of CYP2E1 (48, 49), another P450 susceptible to irreversible heme-modification, argues that redox flux can significantly contribute to P450 turnover. This combined indictment of redox flux notwithstanding, it is also conceivable that other relevant proteolytic determinants i.e structural degrons may also contribute towards this CYP3A UPD-proclivity, as heterologously expressed CYP3A4 is degraded via UPD in yeast in spite of minimal redox flux (26). The potential existence of CYP3A degrons is further underscored by the finding that the appendage of CYP3A4 C-terminal heptapeptide onto the C-terminus of CYP2B1 is sufficient to divert this P450 from ALD to UPD, without altering its structure or function (26). Collectively, these findings suggest that CYP3A redox function coupled with its intrinsic proteasomal degrons, significantly contributes to its inherent Ubdependent proteasomal susceptibility. However, when this redox function is aborted by TAOcomplexation, alternative pathways such as ALD may be coopted for CYP3A degradation.

We have previously identified several enzymes/proteins involved in the UPD of heterologously expressed native CYP3A4 in S. cerevisiae, using well characterized and validated mutant yeast strains genetically deficient in these proteins and corresponding isogenic wild type yeast (25, 26). These studies have identified several bona fide ERAD components such as Cue1p/Ubc7p (E2), the 19S proteasomal lid component Hrd2p, and the AAA ATPase Cdc48p/Ufd1p/Hrd4p complex, while excluding others such as the Ub-ligases (E3s) Hrd1p/Hrd3p, Doa10p and Rsp5p, well recognized participants in ERAD of several other integral and lumenal ER proteins as well as soluble nuclear/cytosolic proteins $\left(50^{-54}\right)$. To identify and characterize the corresponding hepatic proteins participating in CYP3A ERAD/UPD in cultured primary hepatocytes, in vivo/in situ PFA-crosslinking coupled with proteomic analyses was employed. These studies revealed that polyubiquitylated CYPs $3 \mathrm{~A}$ were associated with the mammalian 
Cdc48p homolog, p97. These CYP3A-p97 interactions were enhanced by DDEP-mediated CYP3A inactivation and consequent UPD enhancement, and further magnified by MG-262elicited proteasomal inhibition. Consistent with these PFA-crosslinking/proteomic analyses, CIFM analyses confirmed that morphologically p97 was truly associated in vivo with CYP3A, and these interactions were also enhanced by DDEP inactivation and further magnified by MG-262. These findings thus clearly indicated that as in the case of CYP3A4 in $S$. cerevisiae, CYP3A ERAD in cultured rat hepatocytes also involves the participation of the AAA ATPase p97, the mammalian homolog of Cdc48p.

p97 (aka VCP, valosin-containing protein and its yeast homolog Cdc48p) is an abundant cytosolic AAA ATPase required for numerous cellular processes including ER protein degradation $\left(28^{-35}\right)$. It functions as a heteromeric complex in concert with its cofactors Ufd1p and Npl4/Hrd4p, which bind to its $\mathrm{N}$-terminal domain $\left(28^{-35}\right)$. Ufd1p and Npl4p recruit polyubiquitylated target substrates to the p97-complex by engaging polyUb chains $\left(28^{-35}\right)$. This p97 complex is required for the translocation and/or extraction of ubiquitylated integral and lumenal ER-proteins from the ER-membrane into the cytosol and their subsequent delivery to the $26 \mathrm{~S}$ proteasome $\left(28^{-35}\right)$. p97 is proposed to: (i) act as a "segregase" that recognizes and segregates ubiquitylated from non-ubiquitylated proteins at the ER membrane, thus marking them for proteasomal degradation; and (ii) serve as a "dislocase" that directly exerts force through ATP hydrolysis to extract the target protein out of/across the ER-membrane before delivery to the cytosolic proteasome $(4,33,35)$. Although it has also been proposed to recruit an Ub-ligase to the ER-membrane-anchored target (33), more recent findings on its interaction with the ER-membrane anchored Ub-ligase gp78 reveal that it may itself be recruited to the ER-membrane by this E3 enzyme (55). Apparently, in mammalian systems, a C-terminal 30residue gp78 domain containing a conserved p97/VCP-interacting motif (VIM), selectively recruits p97 from the cytosol to the ER by engaging its N-terminal ND1 (1-470 residues) domain (55). At the ER p97 can interact with at the least four different ER-membrane anchored proteins: p97/VCP-interacting membrane protein (VIMP), Derlin-1, Derlin-2, and an ERADassociated ER-anchored E3 (gp78 or HRD1) (55). The precise functional role of p97 in CYP3A ERAD is unclear given that P450s although tethered to the ER-membrane, are extensively exposed to the cytosol and thus readily accessible to the cytosolic ubiquitylation machinery and/or 26S proteasome. Although admittedly, our p97 studies in hepatocytes document only its direct association with ubiquitylated CYP3A during this process, taken together with our findings of impaired CYP3A4 degradation in $c d c 48 / u f d 1 / h r d 4$-deficient yeast (26), they provide compelling evidence for the functional participation of p97 in CYP3A ERAD, wherein it conceivably could play both of the above roles.

However, although the PFA-crosslinking/proteomic analyses of these immunoprecipitated complexes from cultured rat hepatocytes amply verified that the p97-associated CYP3A was polyubiquitylated, we were unable to detect either the specific hepatic E2 (Ubc) or the E3 Ubligase involved in this process. A limitation of these analyses is that in spite of the highly purified anti-CYP3A IgGs used in the immunoprecipitation analyses, the non-specific protein background was enormous and swamped the detection of much less abundant and possibly transient and/or weakly interacting proteins such as the E2 and E3 species present at normal catalytic levels. Since immunoprecipitation analyses are incompatible with stringent protein denaturation, the conditions used might also have been inadequate to sufficiently reduce/ eliminate the non-specific protein background. A further limitation might have been the use of trypsin alone rather than in combination with lysylendopeptidase-C to digest possibly hydrophobic/intractable proteins (i.e. an ER polytopic E3 protein).

Nevertheless, collectively our findings reveal that native rat CYPs 3A in cultured primary hepatocytes undergo UPD rather than ALD, just like heterologously expressed native human liver CYP3A4 in S. cerevisiae. This CYP3A UPD in hepatocytes as well as yeast is directly 
associated with the p97/Cdc48p ATPase chaperone complex, thereby providing further direct validation of the yeast model for characterization of mammalian liver P450 degradation pathways.

\section{Supplementary Material}

Refer to Web version on PubMed Central for supplementary material.

\section{Acknowledgments}

We wish to sincerely thank Drs T.S. Benedict Yen and Juan Engel, co-Directors of the UCSF Liver Center Microscopy and Advanced Imaging Core for the CIFM analyses, supported by NIH grant P30DK26743. We also acknowledge the use of the UCSF Mass Spectrometry Facility (A.L. Burlingame, Director) supported by the Biomedical Research Technology Program of the National Center for Research Resources, NIH NCRR RR01614 and P30DK26743, as well as sincerely thank Mr. David Maltby for his generous assistance in some of these proteomic analyses.

\section{REFERENCES}

1. Correia MA. Hepatic cytochrome P450 degradation: mechanistic diversity of the cellular sanitation brigade. Drug Metab Rev 2003;35:107-143. [PubMed: 12959413] and references therein

2. Klionsky DJ. The molecular machinery of autophagy: unanswered questions. J Cell Sci 2005;118:718. [PubMed: 15615779]

3. Ohsumi, Y. Molecular dissection of autophagy in the yeast Saccharomyces cerevisiae.. In: Mayer, RJ.; Ciechanover, A.; Rechsteiner, M., editors. Protein degradation. 2. The Ubiquitin-Proteasome system; 2006. 2006. p. 31-50.

4. Romisch K. Endoplasmic reticulum-associated degradation. Annu Rev Cell Dev Biol 2005;21:435456. [PubMed: 16212502]

5. Schafer A, Wolf DH. Endoplasmic reticulum-associated protein quality control and degradation: genome-wide screen for ERAD components. Methods Mol Biol 2005;301:289-292. [PubMed: 15917640]

6. Hampton RY. ER-associated degradation in protein quality control and cellular regulation. Curr Opin Cell Biol 2002;14:476-482. [PubMed: 12383799]

7. Masaki R, Yamamoto A, Tashiro Y. Cytochrome P-450 and NADPH-cytochrome P-450 reductase are degraded in the autolysosomes in rat liver. J Cell Biol 1987;104:1207-1215. [PubMed: 3106362]

8. Ronis MJ, Johansson I, Hultenby K, Lagercrantz J, Glaumann H, Ingelman-Sundberg M. Acetoneregulated synthesis and degradation of cytochrome P450E1 and cytochrome P4502B1 in rat liver [corrected]. Eur J Biochem 1991;198:383-389. [PubMed: 2040300]

9. Roberts BJ, Song BJ, Soh Y, Park SS, Shoaf SE. Ethanol induces CYP2E1 by protein stabilization. Role of ubiquitin conjugation in the rapid degradation of CYP2E1. J Biol Chem 1995;270:2963229635. [PubMed: 8530344]

10. Bardag-Gorce F, Li J, French BA, French SW. Ethanol withdrawal induced CYP2E1 degradation in vivo, blocked by proteasomal inhibitor PS-341. Free Radic Biol Med 2002;32:17-21. [PubMed: 11755313]

11. Murray BP, Zgoda VG, Correia MA. Native CYP2C11: heterologous expression in Saccharomyces cerevisiae reveals a role for vacuolar proteases rather than the proteasome system in the degradation of this endoplasmic reticulum protein. Mol Pharmacol 2002;61:1146-1153. [PubMed: 11961133]

12. Liao M, Zgoda VG, Murray BP, Correia MA. Vacuolar degradation of rat liver CYP2B1 in Saccharomyces cerevisiae: further validation of the yeast model and structural implications for the degradation of mammalian endoplasmic reticulum P450 proteins. Mol Pharmacol 2005;67:14601469. [PubMed: 15703377]

13. Correia MA, Decker C, Sugiyama K, Caldera P, Bornheim L, Wrighton SA, Rettie AE, Trager WF. Degradation of rat hepatic cytochrome P-450 heme by 3,5-dicarbethoxy-2,6-dimethyl-4-ethyl-1,4dihydropyridine to irreversibly bound protein adducts. Arch Biochem Biophys 1987;258:436-451. [PubMed: 3674884] 
14. Correia MA, Sugiyama K, Yao KQ. Degradation of rat hepatic cytochrome P-450p. Drug Metab Rev 1989;20:615-628. [PubMed: 2680399]

15. Sohn DH, Yun YP, Park KS, Veech RL, Song BJ. Post-translational reduction of cytochrome P450IIE by CCl4, its substrate. Biochem Biophys Res Commun 1991;179:449-454. [PubMed: 1883371]

16. Tierney DJ, Haas AL, Koop DR. Degradation of cytochrome P450 2E1: selective loss after labilization of the enzyme. Arch Biochem Biophys 1992;293:9-16. [PubMed: 1309987]

17. Correia MA, Yao K, Wrighton SA, Waxman DJ, Rettie AE. Differential apoprotein loss of rat liver cytochromes P450 after their inactivation by 3,5-dicarbethoxy-2,6-dimethyl-4-ethyl-1,4dihydropyridine: a case for distinct proteolytic mechanisms? Arch Biochem Biophys 1992;294:493503. [PubMed: 1567205]

18. Correia MA, Davoll SH, Wrighton SA, Thomas PE. Degradation of rat liver cytochromes P450 3A after their inactivation by 3,5-dicarbethoxy-2,6-dimethyl-4-ethyl-1,4-dihydropyridine: characterization of the proteolytic system. Arch Biochem Biophys 1992;297:228-238. [PubMed: 1497342]

19. Dai Y, Cederbaum AI. Inactivation and degradation of human cytochrome P4502E1 by CCl4 in a transfected HepG2 cell line. J Pharmacol Exp Ther 1995;275:1614-1622. [PubMed: 8531136]

20. Lown KS, Bailey DG, Fontana RJ, Janardan SK, Adair CH, Fortlage LA, Brown MB, Guo W, Watkins PB. Grapefruit juice increases felodipine oral availability in humans by decreasing intestinal CYP3A protein expression. J Clin Invest 1997;99:2545-2553. [PubMed: 9153299]

21. Roberts BJ. Evidence of proteasome-mediated cytochrome P-450 degradation. J Biol Chem 1997;272:9771-9778. [PubMed: 9092510]

22. Korsmeyer KK, Davoll S, Figueiredo-Pereira ME, Correia MA. Proteolytic degradation of hememodified hepatic cytochromes P450: A role for phosphorylation, ubiquitination, and the 26S proteasome? Arch Biochem Biophys 1999;365:31-44. [PubMed: 10222036]

23. Wang HF, Figueiredo Pereira ME, Correia MA. Cytochrome P450 3A degradation in isolated rat hepatocytes: 26S proteasome inhibitors as probes. Arch Biochem Biophys 1999;365:45-53. [PubMed: 10222037]

24. Kakar SM, Paine MF, Stewart PW, Watkins PB. 6'7'-Dihydroxybergamottin contributes to the grapefruit juice effect. Clin Pharmacol Ther 2004;75:569-579. [PubMed: 15179411]

25. Murray BP, Correia MA. Ubiquitin-dependent 26S proteasomal pathway: a role in the degradation of native human liver CYP3A4 expressed in Saccharomyces cerevisiae? Arch Biochem Biophys 2001;393:106-116. [PubMed: 11516167]

26. Liao M, Faouzi S, Karyakin A, Correia MA. Endoplasmic reticulum-associated degradation of cytochrome P450 CYP3A4 in Saccharomyces cerevisiae: further characterization of cellular participants and structural determinants. Mol Pharmacol 2006;69:1897-1904. [PubMed: 16556771]

27. Wolf DH. From lysosome to proteasome: the power of yeast in the dissection of proteinase function in cellular regulation and waste disposal. Cell Mol Life Sci 2004;61:1601-1614. [PubMed: 15224185]

28. Bar-Nun S. The role of $\mathrm{p} 97 / \mathrm{Cdc} 48 \mathrm{p}$ in endoplasmic reticulum-associated degradation: from the immune system to yeast. Curr Top Microbiol Immunol 2005;300:95-125. [PubMed: 16573238]

29. Meyer HH, Shorter JG, Seemann J, Pappin D, Warren G. A complex of mammalian ufd1 and npl4 links the AAA-ATPase, p97, to ubiquitin and nuclear transport pathways. Embo J 2000;19:21812192. [PubMed: 10811609]

30. Bays NW, Wilhovsky SK, Goradia A, Hodgkiss-Harlow K, Hampton RY. HRD4/NPL4 is required for the proteasomal processing of ubiquitinated ER proteins. Mol Biol Cell 2001;12:4114-4128. [PubMed: 11739805]

31. Dai RM, Li CC. Valosin-containing protein is a multi-ubiquitin chain-targeting factor required in ubiquitin-proteasome degradation. Nat Cell Biol 2001;3:740-744. [PubMed: 11483959]

32. Rabinovich E, Kerem A, Frohlich KU, Diamant N, Bar-Nun S. AAA-ATPase p97/Cdc48p, a cytosolic chaperone required for endoplasmic reticulum-associated protein degradation. Mol Cell Biol 2002;22:626-634. [PubMed: 11756557]

33. Ye Y, Shibata Y, Kikkert M, van Voorden S, Wiertz E, Rapoport TA. Inaugural Article: Recruitment of the p97 ATPase and ubiquitin ligases to the site of retrotranslocation at the endoplasmic reticulum membrane. Proc Natl Acad Sci U S A 2005;102:14132-14138. [PubMed: 16186510] 
34. Richly H, Rape M, Braun S, Rumpf S, Hoege C, Jentsch S. A series of ubiquitin binding factors connects CDC48/p97 to substrate multiubiquitylation and proteasomal targeting. Cell 2005;120:7384. [PubMed: 15652483]

35. Elkabetz Y, Shapira I, Rabinovich E, Bar-Nun S. Distinct steps in dislocation of luminal endoplasmic reticulum-associated degradation substrates: roles of endoplamic reticulum-bound p97/Cdc48p and proteasome. J Biol Chem 2004;279:3980-3989. [PubMed: 14607830]

36. LeCluyse E, Bullock P, Madan A, Carroll K, Parkinson A. Influence of extracellular matrix overlay and medium formulation on the induction of cytochrome P-450 2B enzymes in primary cultures of rat hepatocytes. Drug Metab Dispos 1999;27:909-915. [PubMed: 10421618]

37. Kocarek TA, Schuetz EG, Guzelian PS. Biphasic regulation of cytochrome P450 2B1/2 mRNA expression by dexamethasone in primary cultures of adult rat hepatocytes maintained on matrigel. Biochem Pharmacol 1994;48:1815-1822. [PubMed: 7980651]

38. Han XM, Lee G, Hefner C, Maher JJ, Correia MA. Heme-reversible impairment of CYP2B1/2 induction in heme-depleted rat hepatocytes in primary culture: translational control by a hepatic alpha-subunit of the eukaryotic initiation factor kinase? J Pharmacol Exp Ther 2005;314:128-138. [PubMed: 15769864]

39. Ortiz de Montellano PR, Beilan HS, Kunze KL. N-Alkylprotoporphyrin IX formation in 3,5dicarbethoxy-1,4-dihydrocollidine-treated rats. Transfer of the alkyl group from the substrate to the porphyrin. J Biol Chem 1981;256:6708-6713. [PubMed: 6894597]

40. Irving MG, Roll FJ, Huang S, Bissell DM. Characterization and culture of sinusoidal endothelium from normal rat liver: lipoprotein uptake and collagen phenotype. Gastroenterology 1984;87:12331247. [PubMed: 6092194]

41. Vasilescu J, Guo X, Kast J. Identification of protein-protein interactions using in vivo cross-linking and mass spectrometry. Proteomics 2004;4:3845-3854. [PubMed: 15540166]

42. Watkins PB, Wrighton SA, Schuetz EG, Maurel P, Guzelian PS. Macrolide antibiotics inhibit the degradation of the glucocorticoid-responsive cytochrome P-450p in rat hepatocytes in vivo and in primary monolayer culture. J Biol Chem 1986;261:6264-6271. [PubMed: 3486184]

43. Riddick DS, McGilvray I, Marks GS. Inactivation of rat liver microsomal steroid hydroxylations by 4-alkyl analogues of 3,5-diethoxycarbonyl-1,4-dihydro-2,4,6-trimethylpyridine: evidence for selectivity among steroid-inducible cytochrome P450IIIA forms. Can J Physiol Pharmacol 1990;68:1533-1541. [PubMed: 2085799]

44. Eliasson E, Mkrtchian S, Halpert JR, Ingelman-Sundberg M. Substrate-regulated, cAMP-dependent phosphorylation, denaturation, and degradation of glucocorticoid-inducible rat liver cytochrome P450 3A1. J Biol Chem 1994;269:18378-18383. [PubMed: 8034584]

45. Guengerich FP, Johnson WW. Kinetics of ferric cytochrome P450 reduction by NADPH-cytochrome $\mathrm{P} 450$ reductase: rapid reduction in the absence of substrate and variations among cytochrome $\mathrm{P} 450$ systems. Biochemistry 1997;36:14741-14750. [PubMed: 9398194]

46. Denisov IG, Grinkova YV, Baas BJ, Sligar SG. The ferrous-dioxygen intermediate in human cytochrome P450 3A4. Substrate dependence of formation and decay kinetics. J Biol Chem 2006;281:23313-23318. [PubMed: 16762915]

47. Henderson CJ, Otto DM, Carrie D, Magnuson MA, McLaren AW, Rosewell I, Wolf CR. Inactivation of the hepatic cytochrome $\mathrm{P} 450$ system by conditional deletion of hepatic cytochrome $\mathrm{P} 450$ reductase. J Biol Chem 2003;278:13480-13486. [PubMed: 12566435]

48. Goasduff T, Cederbaum AI. NADPH-dependent microsomal electron transfer increases degradation of CYP2E1 by the proteasome complex: role of reactive oxygen species. Arch Biochem Biophys 1999;370:258-270. [PubMed: 10510285]

49. Zhukov A, Ingelman-Sundberg M. Relationship between cytochrome P450 catalytic cycling and stability: fast degradation of ethanol-inducible cytochrome P450 2E1 (CYP2E1) in hepatoma cells is abolished by inactivation of its electron donor NADPH-cytochrome $\mathrm{P} 450$ reductase. Biochem $\mathrm{J}$ 1999;340(Pt 2):453-458. [PubMed: 10333489]

50. Gardner RG, Shearer AG, Hampton RY. In vivo action of the HRD ubiquitin ligase complex: mechanisms of endoplasmic reticulum quality control and sterol regulation. Mol Cell Biol 2001;21:4276-4291. [PubMed: 11390656] 
51. Wilhovsky S, Gardner R, Hampton R. HRD gene dependence of endoplasmic reticulum-associated degradation. Mol Biol Cell 2000;11:1697-1708. [PubMed: 10793145]

52. Swanson R, Locher M, Hochstrasser M. A conserved ubiquitin ligase of the nuclear envelope/ endoplasmic reticulum that functions in both ER-associated and Matalpha2 repressor degradation. Genes Dev 2001;15:2660-2674. [PubMed: 11641273]

53. Hochstrasser M, Papa FR, Chen P, Swaminathan S, Johnson P, Stillman L, Amerik AY, Li SJ. The DOA pathway: studies on the functions and mechanisms of ubiquitin-dependent protein degradation in the yeast Saccharomyces cerevisiae. Cold Spring Harb Symp Quant Biol 1995;60:503-513. [PubMed: 8824423]

54. Haynes CM, Caldwell S, Cooper AA. An HRD/DER-independent ER quality control mechanism involves Rsp5p-dependent ubiquitination and ER-Golgi transport. J Cell Biol 2002;158:91-101. [PubMed: 12105183]

55. Ballar P, Shen Y, Yang H, Fang S. The role of a novel p97/valosin-containing protein-interacting motif of gp78 in endoplasmic reticulum-associated degradation. J Biol Chem 2006;281:35359_ 35368. [PubMed: 16987818]

56. Komori M, Oda Y. A major glucocorticoid-inducible P450 in rat liver is not P450 3A1. J Biochem 1994;116:114-120. [PubMed: 7528203]

57. Mahnke A, Strotkamp D, Roos PH, Hanstein WG, Chabot GG, Nef P. Expression and inducibility of cytochrome P450 3A9 (CYP3A9) and other members of the CYP3A subfamily in rat liver. Arch Biochem Biophys 1997;337:62-68. [PubMed: 8990268]

58. Ovaa, H.; Overkleeft, H.; Kessler, B.; Ploegh, HL. Dissecting intracellular proteolysis using small molecule inhibitors and molecular probes.. In: Mayer, RJ.; Ciechanover, A.; Rechsteiner, M., editors. Protein Degradation. 2. Wiley-CH; 2006. p. 51-78.

59. Zangar RC, Kocarek TA, Shen S, Bollinger N, Dahn MS, Lee DW. Suppression of cytochrome P450 3A protein levels by proteasome inhibitors. J Pharmacol Exp Ther 2003;305:872-879. [PubMed: 12626652]

60. Noreault-Conti TL, Jacobs JM, Trask HW, Wrighton SA, Sinclair JF, Nichols RC. Effect of proteasome inhibition on toxicity and CYP3A23 induction in cultured rat hepatocytes: comparison with arsenite. Toxicol Appl Pharmacol 2006;217:245-251. [PubMed: 17083955] 


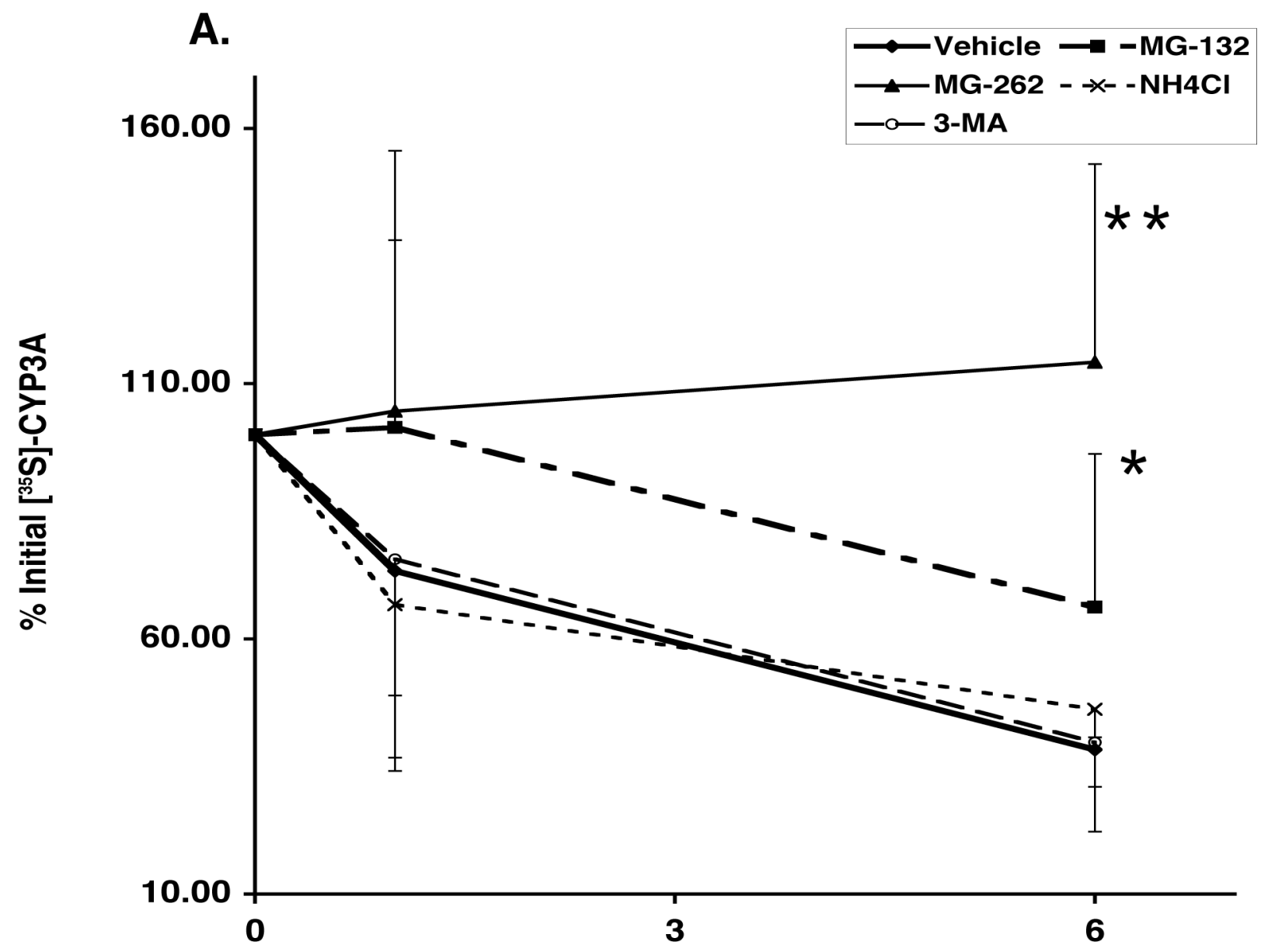

B.

Time (h)

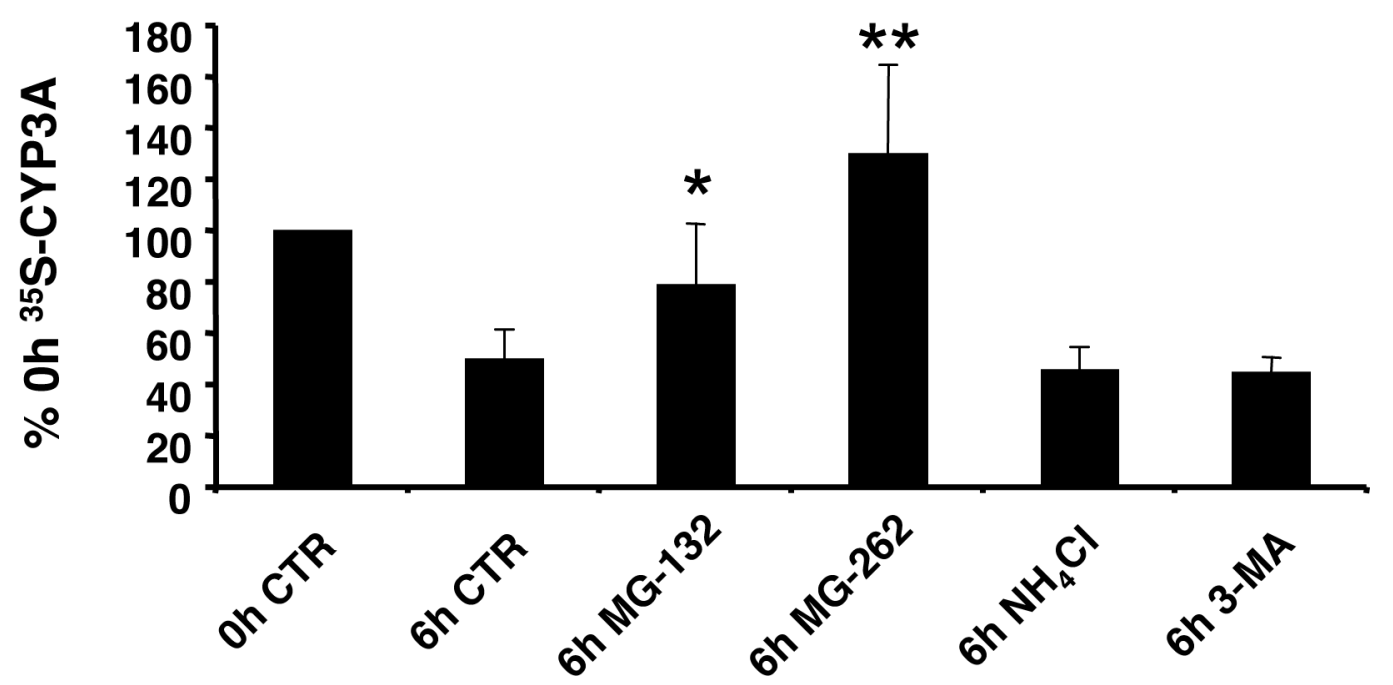




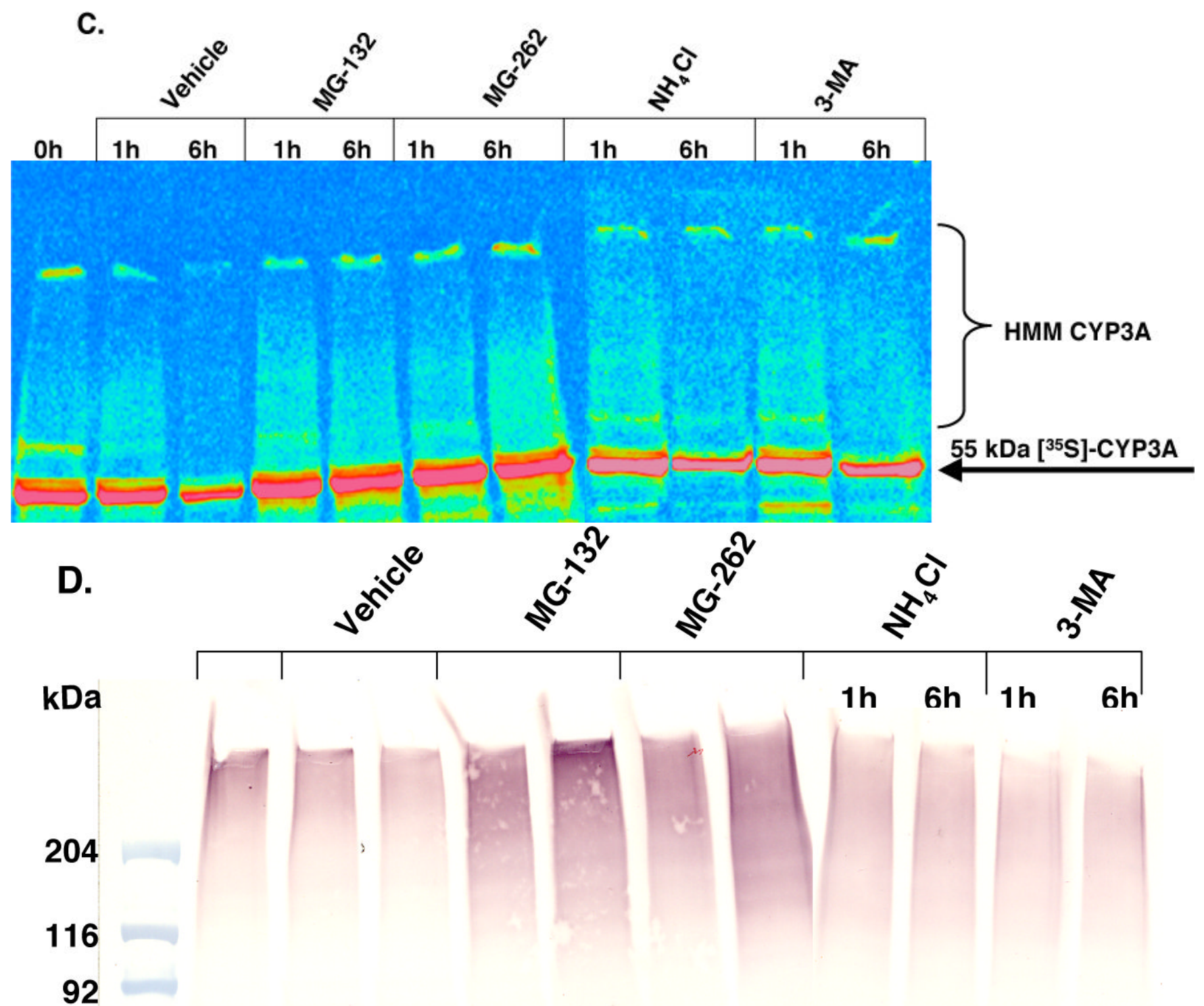

Fig. 1. Turnover of native CYP3A in cultured rat hepatocytes over $6 \mathrm{~h}$

Hepatocytes were cultured, Dex-pretreated and pulse-labeled with ${ }^{35} \mathrm{~S}$-Met/Cys as described (Methods). One h after pulse labeling, MG-132 (20 $\mu \mathrm{M}), \mathrm{MG}-262(10 \mu \mathrm{M}), 3-\mathrm{MA}(5 \mathrm{mM})$ and/or $\mathrm{NH}_{4} \mathrm{Cl}(20 \mathrm{mM})$ were added. Cells were harvested at indicated times thereafter, lysates were prepared and subjected to CYP3A IP analyses. A. Pulse-chase analyses of native CYP3A immunoprecipitates subjected to SDS-PAGE on 4-20\% polyacrylamide gels followed by PhosphorImager and ImageQuant analyses. Values are mean \pm SD obtained from 4-8 individual experiments. At $6 \mathrm{~h}$, statistically significant differences at $\mathrm{p}<0.001$ (**) and $\mathrm{p}<0.05$ 
(*) were found between MG-262- and MG-132-treated values and corresponding vehiclecontrols, respectively. B. Relative CYP3A losses (Mean \pm SD) monitored by ${ }^{35}$ S-radioctivity in CYP3A immunoprecipitates at $6 \mathrm{~h}$ relative to $0 \mathrm{~h}$-control in 5 individual experiments.

Statistically significant differences at $\mathrm{p}<0.01(* *)$ and $\mathrm{p}<0.05(*)$ were found between MG-262and MG-132-treated values and corresponding vehicle-controls, respectively. C. Effects of proteasomal and ALD inhibitors on native CYP3A ubiquitylation (HMM), as monitored by SDS-PAGE and relative ${ }^{35}$ S-fluorography of CYP3A immunoprecipitates by PhosphorImager analyses from cells harvested at 0,1 and $6 \mathrm{~h}$ post-chase. The relative intensity of the ${ }^{35} \mathrm{~S}$ -

labeling according to the color wheel intensity code is: white $>$ red $>$ orange $>$ yellow $>$ green $>$ blue. D. Corresponding native CYP3A ubiquitylation profiles as determined by Western IB analyses of CYP3A immunoprecipitates with anti-Ub IgGs as described (Methods). 


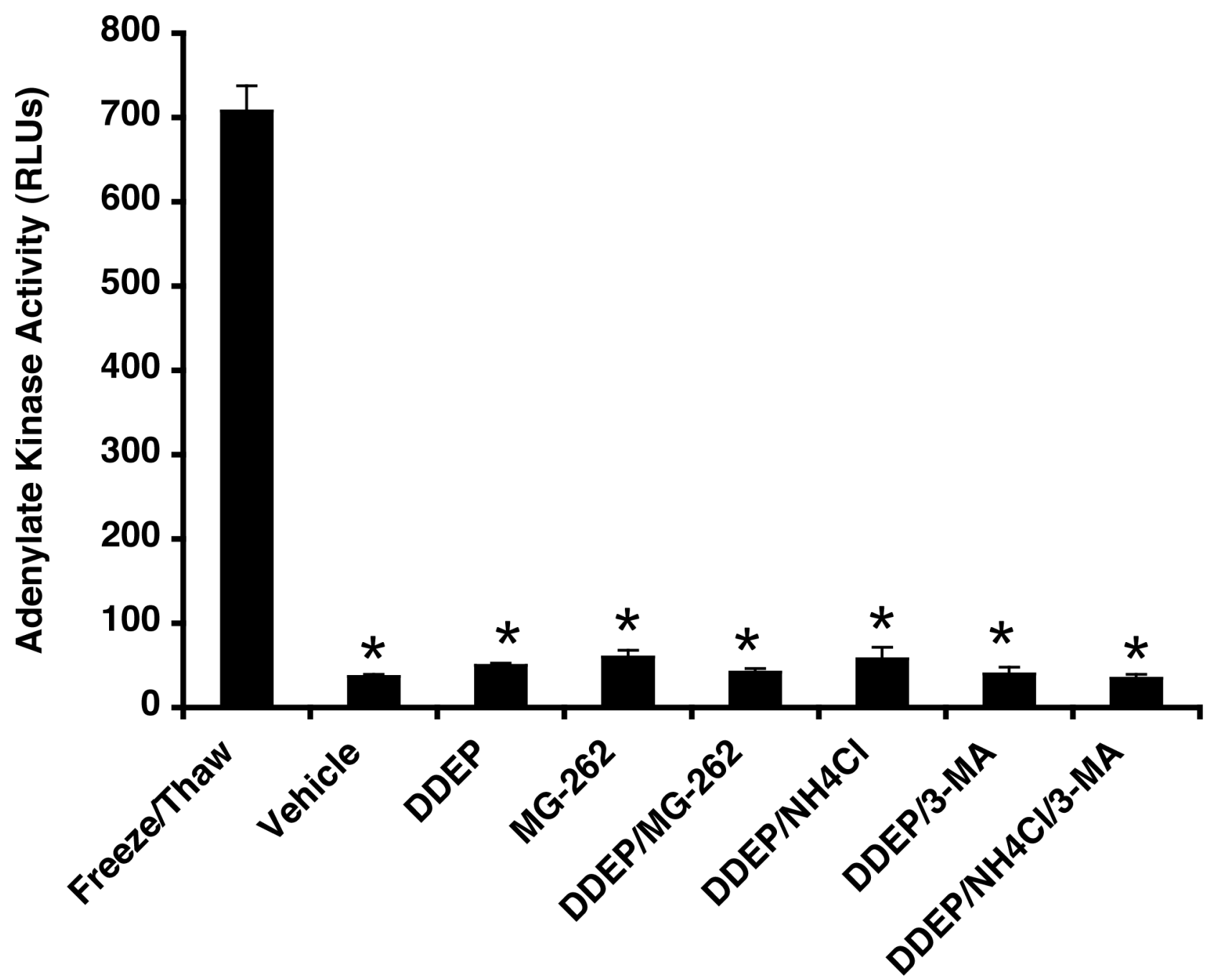

Fig. 2. Assessment of relative cytotoxicity profiles of cultured hepatocytes after treatment with DDEP and/or ALD or proteasomal inhibitors

Cells were treated with DDEP in the presence or absence of ALD or proteasomal inhibitors as described (Methods). At $6 \mathrm{~h}$ after DDEP treatment, media from the variously treated cell cultures were collected and assayed for AK activity by the ToxiLight protocol. Values were expressed as relative luminescence units (RLUs; mean $\pm \mathrm{SD} ; n=3$ ), and compared with a positive control consisting of AK released by damage inflicted by repeated cycles of freeze thawing. Asterisks indicate statistically significant differences at $p<0.001$ from freeze-thawed AK-values (positive control). None of the treatments resulted in any statistically significant leakage of AK into the culture medium relative to the vehicle control. 
A.

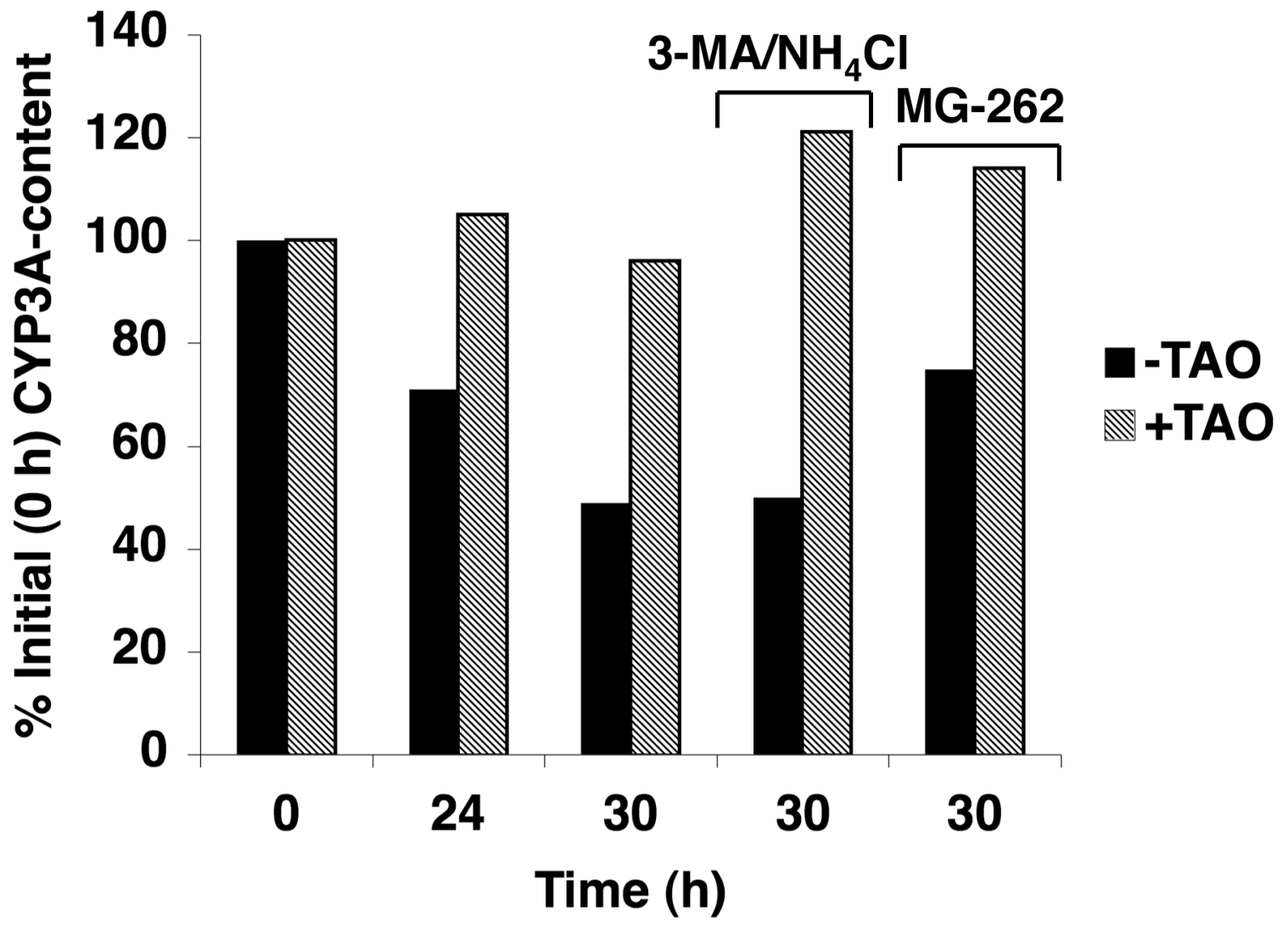


C.

kDa

204

116

92

\section{Time (h):}

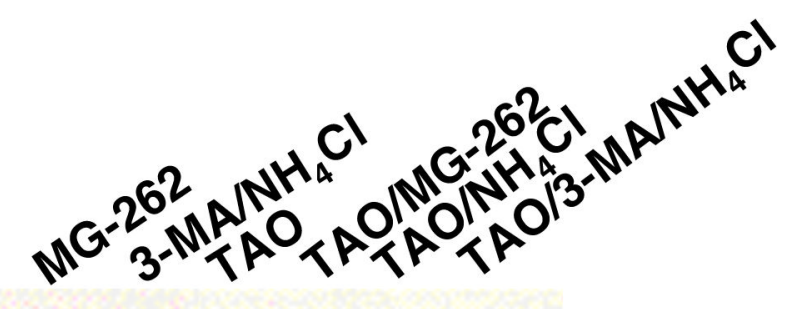

C'

Fig. 3. Effects of TAO-treatment and/or ALD or proteasomal inhibitors on CYP3A stability in cultured hepatocytes

Cells were treated with TAO in the presence or absence of ALD or proteasomal inhibitors as described (Methods). A. Microsomes were prepared from cells harvested at the indicated times and subjected to IB analyses with anti-CYP3A IgGs. B. Corresponding densitometric quantitation of immunoblots from microsomes pooled from two separate cell cultures in two separate experiments. MG-262 and 3MA/ $\mathrm{NH}_{4} \mathrm{Cl}$ were present during the last $6 \mathrm{~h}$ of $30 \mathrm{~h}$ TAOtreatment. C. Corresponding CYP3A ubiquitylation profiles obtained by Western IB analyses of CYP3A immunoprecipitates with anti-Ub IgGs as described (Methods). 

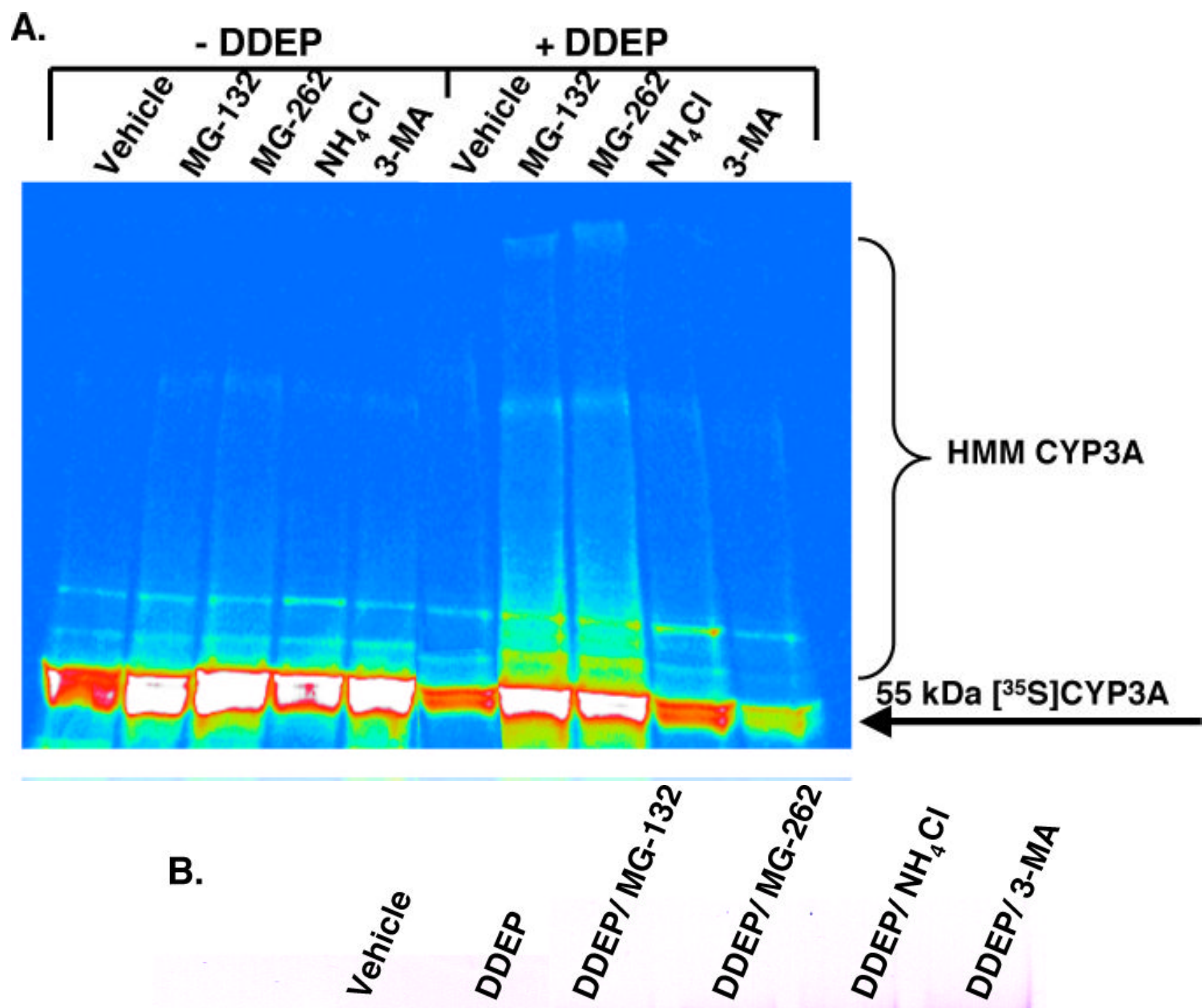

kDa

204

116

92

50

37

Biochemistry. Author manuscript; available in PMC 2008 September 15. 
Fig. 4. Effects of proteasomal and ALD inhibitors on native (-DDEP) and DDEP-inactivated CYP3A turnover

Cells were ${ }^{35} \mathrm{~S}$-pulse-labeled as described (Methods). At 0 post-chase, cells were treated with $\operatorname{DDEP}(100 \mu \mathrm{M})$ or equivalent volume of vehicle (DMSO) in the presence or absence of proteasomal or ALD inhibitors and harvested $6 \mathrm{~h}$ later. A. Effects of UPD and ALD inhibitors on native and DDEP-inactivated CYP3A loss (detected at $55 \mathrm{kDa}$ ) and ubiquitylation (HMM), as monitored by SDS-PAGE and relative ${ }^{35}$ S-fluorography of CYP3A immunoprecipitates by PhosphorImager analyses. The relative intensity of the ${ }^{35} \mathrm{~S}$-labeling according to the color wheel intensity code is: white $>$ red $>$ orange $>$ yellow $>$ green $>$ blue. B. Corresponding native or DDEP-inactivated CYP3A ubiquitylation profiles as determined by Western IB analyses of CYP3A immunoprecipitates with anti-Ub IgGs as described (Methods). 


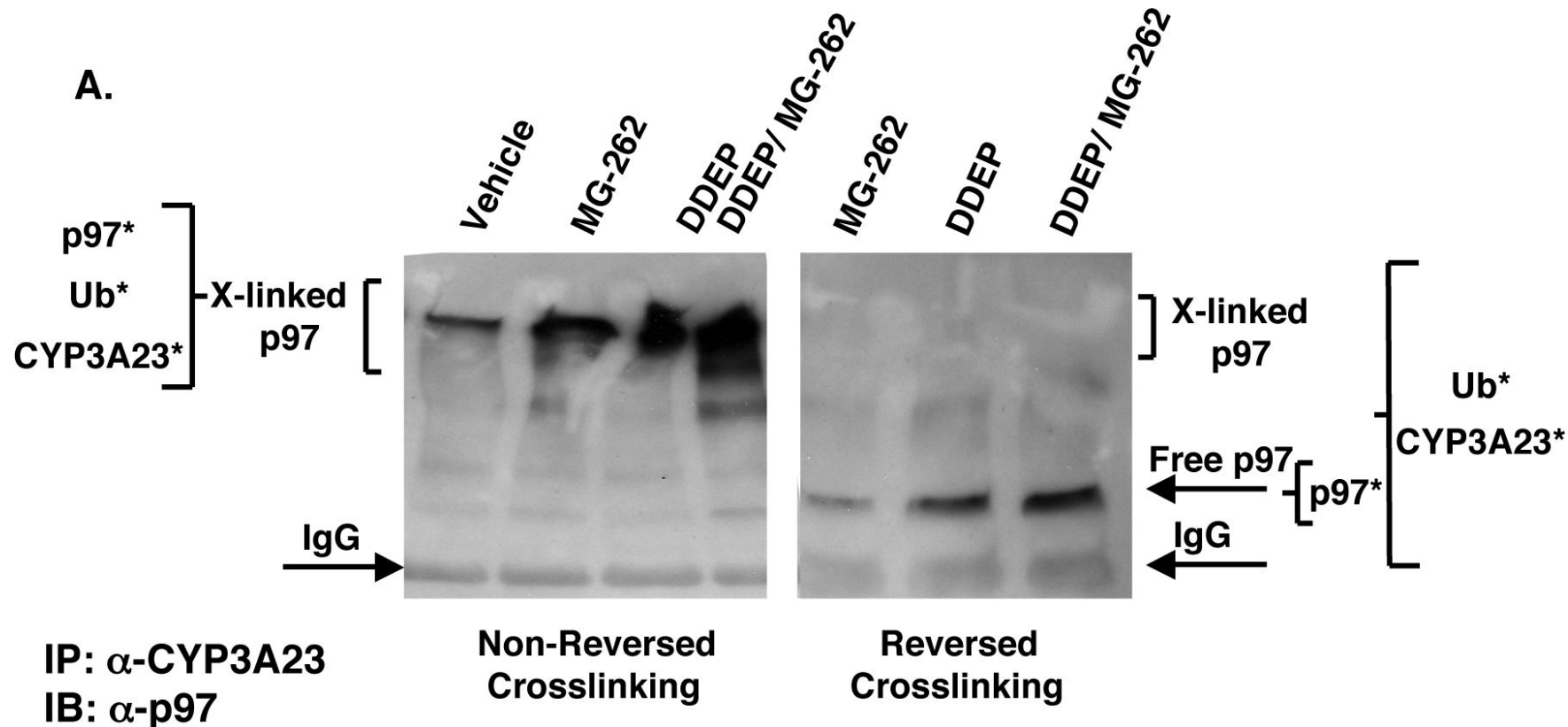

B.

CYP 3A
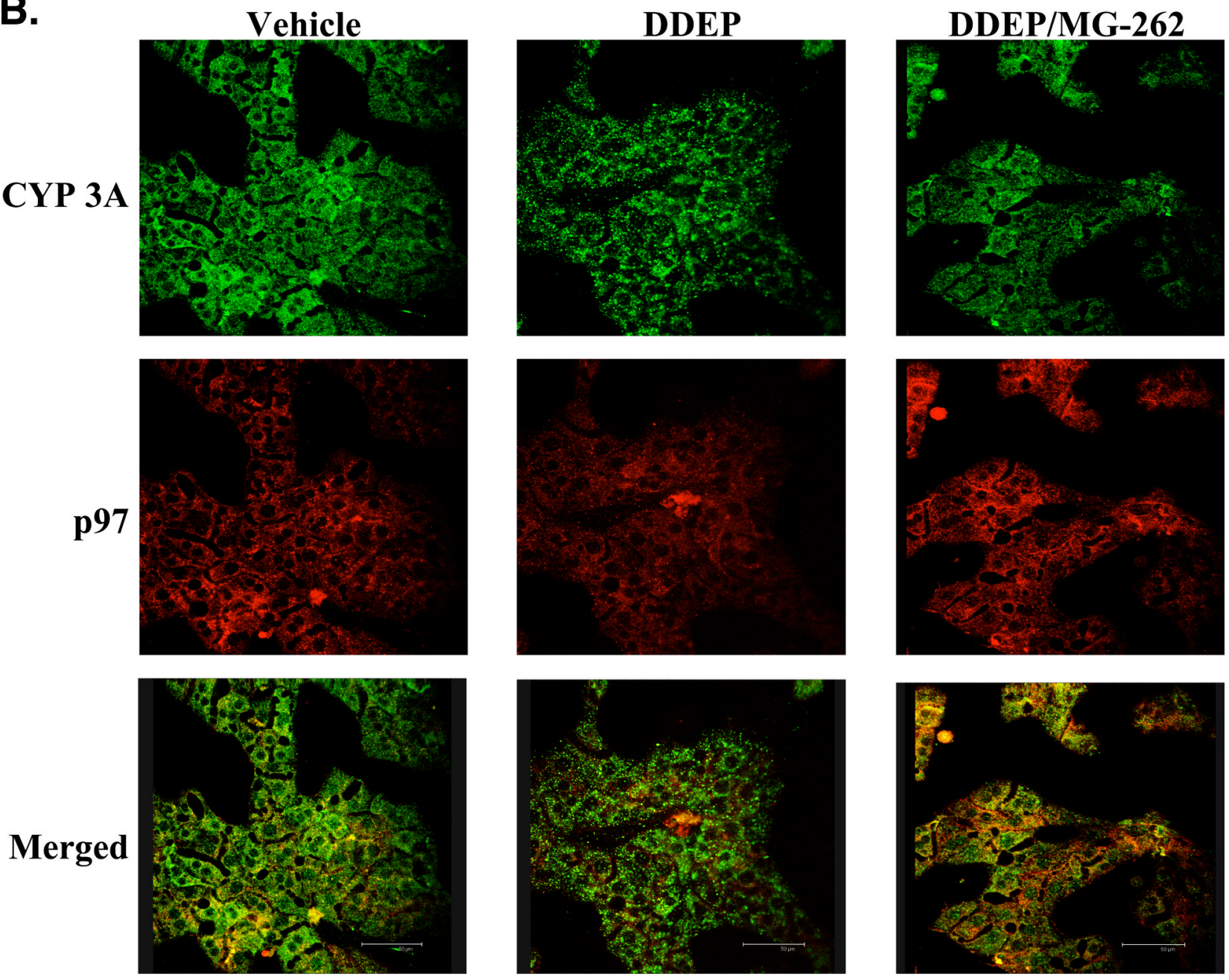
Fig. 5. p97-CYP3A interaction during CYP3A ERAD through PFA crosslinking/proteomic and CIFM analyses

A. p97 IB analyses of in situ PFA-crosslinked CYP3A immunoprecipitates: Cultured rat hepatocytes were treated with or without DDEP and/or MG-262 for 0-1 h, and in vivo crosslinked with $2.5 \%$ PFA for $15 \mathrm{~min}$ at $37^{\circ} \mathrm{C}$. After termination, cells were harvested and CYP3A immunoprecipitates were subjected to p97 IB analyses before (a) or after reversal of crosslinking by boiling at $95^{\circ} \mathrm{C}$ for $10 \mathrm{~min}(\mathbf{b})$. In parallel, CYP3A coimmunoprecipitates were also subjected to SDS-PAGE, sequential slicing of the gels and in situ tryptic digestion of gel bands followed by LC-MS/MS analyses of tryptic peptides by reversed-phase HPLC (Methods). The copresence of ubiquitylated CYP3A23 and p97 in these CYP3A immunoprecipitates was confirmed by LC-MS/MS analyses of the in situ tryptic digests of gel bands from both $a$ and $b$. As indicated by the asterisks, in (a) ubiquitylated CYP3A comigrated as the crosslinked p97 complex to the top of the gel (the presence of $\mathrm{Ub}$ was confirmed from 7 different CID spectra with a $71 \%$ sequence coverage; p97 was identified from its acetylated $\mathrm{N}$-terminal peptide, but 2 CID spectra acquired from the $2+$ as well as the $3+$ molecular ions; and CYP3A1 was identified from 3 unique peptides - Supplementary Figures 1-3 illustrate the data quality). After PFA-crosslinking reversal (b) ubiquitylated CYP3A and p97

electrophoretically migrated according to their relative masses. No corresponding ions were found in tryptic digests of the mock immunoprecipitation controls, run in parallel. The electrophoretic migration profiles of CYP3A and ubiquitin were consistent with that of ${ }^{35} \mathrm{~S}$ CYP3A HMM (Fig. 4A) and ubiquitylated CYP3A (Fig. 4B).

B. p97-Colocalization with CYP3A by CIFM analysis in untreated, DDEP- and DDEP/ MG-262-treated rat hepatocytes: After crosslinking, treated and untreated rat hepatocyte cultures were fixed and simultaneously stained with antibodies to CYP3A (green) and p97 (red). 


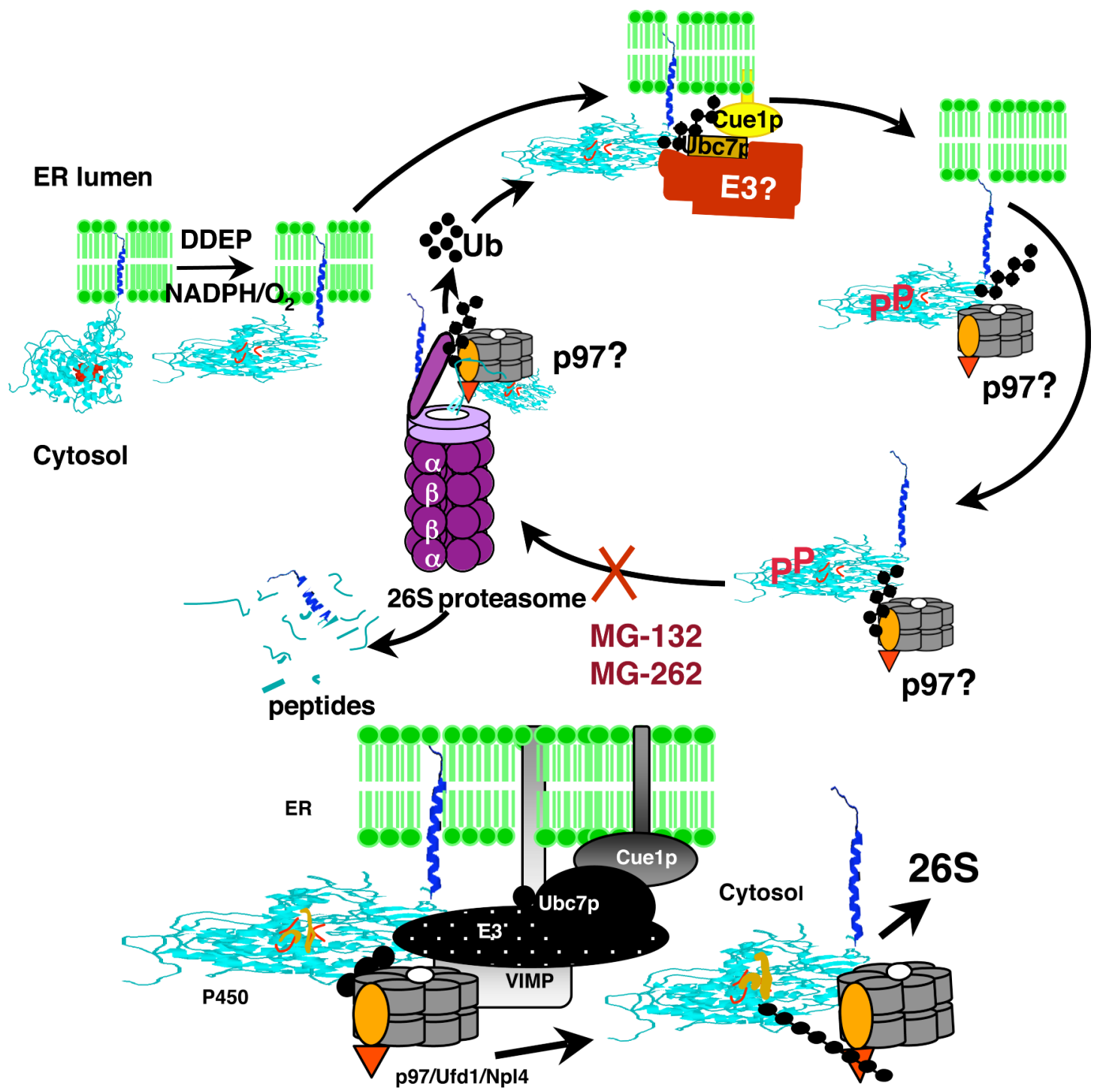

Fig. 6. ERAD/UPD of DDEP-inactivated CYPs 3A

A plausible role for $\mathrm{p} 97$ in the dislocation of ubiquitylated CYP3A and delivery to the $26 \mathrm{~S}$ proteasome. 